\title{
EL RESIDUO LÍQUIDO DE LAS CURTIEMBRES ESTUDIO DE CASO: CUENCA ALTA DEL RÍO BOGOTÁ
}

\section{THE LIQUID REMAINDER OF THE TANNERIES CASE STUDY: HIGH RIVER BASIN OF THE BOGOTA RIVER}

Jorge Luis Corredor Rivera ${ }^{1}$

Fecha de Recepción: 13 de Octubre de 2006

Fecha de Aprobación: 16 de Noviembre de 2006

RESUMEN: El tratamiento de pieles de animales para su empleo en la industria marroquinera, del vestido y del calzado, produce residuos líquidos que tienen altas implicaciones nocivas sobre el recurso hídrico. Sobre el tratamiento que debe darse a los residuos líquidos y sólidos provenientes de la industria de las curtiembres se han realizado muchas pruebas y estudios, para optimizar la calidad de la disposición final de los mismos. En este sentido, se comentan algunos de los aspectos físico-químicos de los residuos líquidos relacionados con esta industria y sus efectos sobre el ambiente, así como algunas propuestas para manejarlos con la perspectiva que sean aplicables en el entorno colombiano. Con el fin de dar una idea de tales implicaciones y posibles soluciones, se presenta un somero análisis para la cuenca alta del río Bogotá, en el sector vecino a la cabecera municipal de Villapinzón y el sector de San Benito en la ciudad de Bogotá.

PALABRAS CLAVE: Curtiembre, residuo líquido, tanino, wet-blue, vertimiento

ABSTRACT: The treatment of animal skins for your use in the industry of the leatherwork, clothing and shoes, have noxious high effects over water resources that received this pouring. In order to offer an idea of those effects, this is an analysis quick for the basin height of Bogotá River, in the nearness of Villapinzón city. For the treatment that required the liquid and solid disposal with origin in the leatherwork industry revising many proofs and studies, for optimized the quality of their drainage and solid residuals and final disposition. In this order, talk about it over some aspects physical and chemist of this residuals and their effects over the environment and their handling for the specifically sectors in Colombia. Finally, included some proposal for handle that residuals in order to your application in colombian environment.

KEY WORDS: Tanning, waste water, tannin, wet-blue, shedding

1 Ingeniero Civil, Docente Facultad de Ingeniería, Universidad Militar Nueva Granada, jcorred@umng.edu.co, Bogotá, Colombia 


\section{INTRODUCCIÓN}

La contaminación inducida en las fuentes superficiales proviene de diferentes orígenes de tipo antrópico. La creciente preocupación en la comunidad residente en la cuenca del río Bogotá por su recuperación y manejo, desde hace años ha generado la realización de diferente tipo de estudios y acciones para cumplir con tal cometido. Sin embargo los resultados obtenidos son aún incipientes. En este caso, solamente, se busca cumplir con una función informativa sobre la calidad de los efluentes de los vertimientos líquidos producto de la industria de las curtiembres y sus efectos sobre las aguas naturales superficiales, en particular sobre el río Bogotá.

La industria de cuero contempla un proceso que inicia con el sacrificio y faenado del ganado, para seguir con su desuello, del que se obtiene la piel, la cual es sometida a un proceso de curtición y terminado, y de esta forma entregarla para la fabricación de productos de diversa índole como cinturones, pulsos para relojes, calzado, vestido, maletas, maletines, revestimiento de muebles, tapicería interna de vehículos, sillas de montar y gran variedad de productos de consumo. De esta gran industria, solamente se trata en este análisis la problemática relacionada con los procesos de curtición, teniendo en cuenta que corresponde a la mayor productora de contaminación ambiental.

\section{LAS CURTIEMBRES EN LA CUENCA DEL RÍO BOGOTA}

La producción de cueros con diferentes grados de terminado, se realiza en áreas sobre las que convergen varios factores que el sean favorables, entre ellos: fácil acceso al agua, disponibilidad y costo de materia prima y mano de obra, así como oportunidad de transporte y comercialización.

La práctica del curtido del cuero es muy antigua, en el sector de Villapinzón se conoce desde hace más de 150 años, cuando se utilizaban curtientes vegetales de los árboles nativos, luego hacia los comienzos de la década de los 60 se conocieron los primeros fulones instalados en ambas márgenes de la carretera central del norte. En la década de los 80 se estableció la necesidad de intervenir esta industria debido a los altos efectos contaminantes que estaban produciéndose sobre el río Bogotá, lo cual trajo como consecuencia que algunas de estas curtiembres desplazaran sus actividades a la ciudad de Bogotá, en donde se concentraron en el conocido sector de San Benito, vecino al río Tunjuelito.

En Colombia sobresalen en la industria de las curtiembres: en Cundinamarca (Villapinzón y Bogotá), en Valle (Cartago y Cerritos), en Nariño (Pasto y Belén), en Tolima (Ibagué) y en Quindío (La María), sin descartar las que existen en otras zonas del país como el Valle de Aburrá, Cúcuta, Barranquilla y Cartagena; desde estas tres últimas se transportan pieles crudas a Villapinzón y Bogotá para su procesamientoº ${ }^{2}$. Para este análisis se consideró la información disponible para los sectores de Villapinzón y Bogotá, en particular para el sector de San Benito, en donde se concentra la industria de las curtiembres en la capital del país.

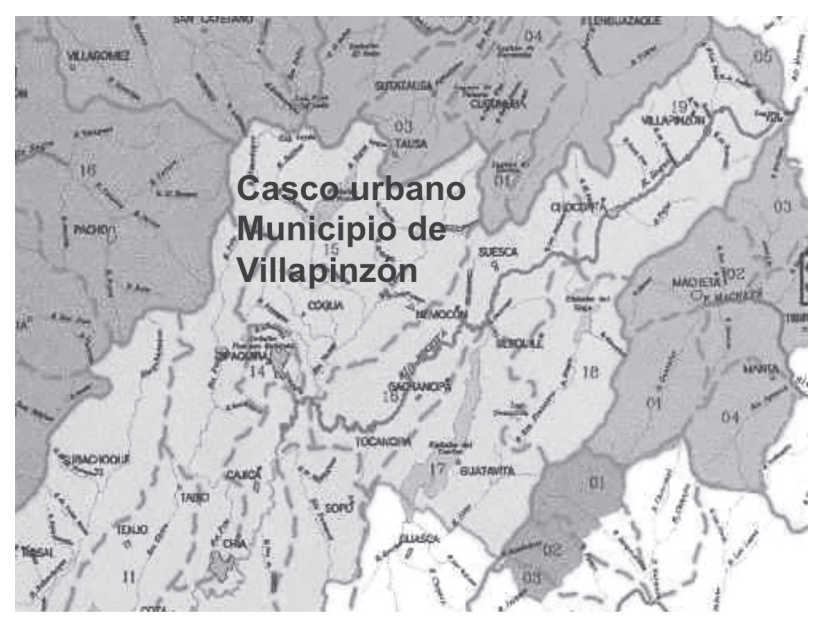

Figura 1. Ubicación del área de influencia

Fuente: Corporación Autónoma Regional Cundinamarca, 2000 
El área de interés para este análisis en el sector circunvecino a la cabecera municipal de Villapinzón en Cundinamarca, se muestra en la Figura 1, pues es allí en donde se instalan las primeras microindustrias en la cuenca del río Bogotá para el tratamiento de pieles de animal, siendo el caso específico las correspondientes al ganado vacuno, industria más conocida como curtiembres, cuyo producto final serán la marroquinería, el vestido y el calzado.

Esta actividad se caracteriza en este sector por ser dominantemente de tipo artesanal, aunque algunas de ellas se pueden calificar como industrializadas. En ellas se tratan pieles provenientes de diferentes partes del país, especialmente de la costa atlántica.

En esta industria se clasifica como pequeña aquella que procesa hasta 100 pieles/mes, mediana hasta $1500 \mathrm{pieles} / \mathrm{mes}$ y grande entre 1500 y 10.000 pieles/mes ${ }^{3}$. Para las pieles frescas provenientes de la Sabana de Bogotá, se encontró que su peso oscila entre 20 y $25 \mathrm{~kg} /$ piel $^{4}$.

En este sector a 2001, se tenían identificadas cerca de 175 industrias de curtiembres ${ }^{5}$. La localización de algunas de ellas se muestra en la Figura 2, en el cual cada curtiembre se identifica con el número del expediente de radicación de permiso de vertimientos ante la CAR-Cundinamarca, aproximadamente.

Informativamente, en 1992, la distribución de las industrias de curtiembres repartidas por veredas en el municipio de Villapinzón, era la que se indica en la Figura 3. (CAR-Cundinamarca)

En forma aproximada, se ha identificado en Colombia 737 curtiembres de las cuales 4 se clasifican como gran empresa, estas procesan en $25 \%$ equivalente a

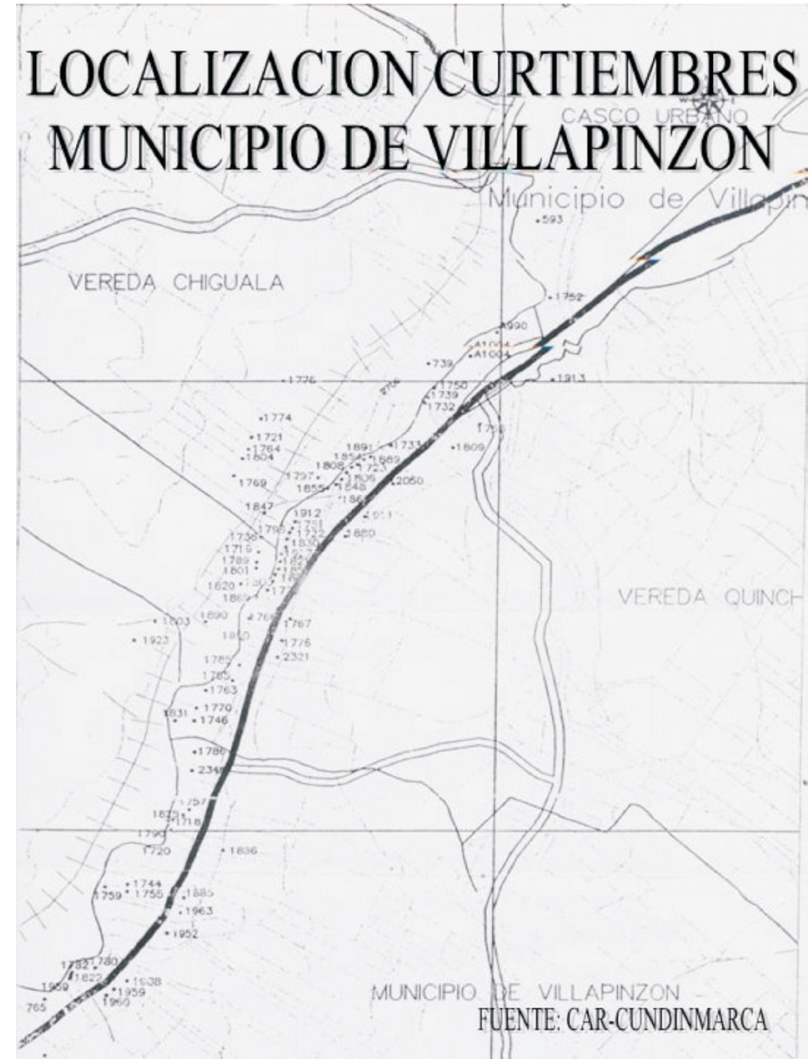

Figura 2. Localización de curtiembres, Villapinzón Fuente: CAR-Cundinamarca

3,5 millones de pieles por año, dentro de la mediana industria se incluyen 8 curtiembres que procesan el $17 \%$ de la producción total , las otras 725 curtiembres tratan el $58 \%$ restante, el cual se encuentra distribuido así: 121 en el municipio de Villapinzón, 50 en el municipio de Chocontá y 9 en el municipio de Cogua, en el entorno de la jurisdicción de la CAR-Cundinamarca y 350 en el sector de San Benito en Bogotá (300 según Ecoparque San Benito), entre el río Tunjuelito y la calle 58 sur y entre la Avenida Tunjuelito y la Avenida Boyacá, el resto de las curtiembres se distribuyen en otras ciudades del país ${ }^{6}$.

3 PLAN DE ORDENAMIENTO TERRITORIAL - POT, Municipio de Villapinzón, 2000

4 ECOPARQUE SAN BENITO, 2001

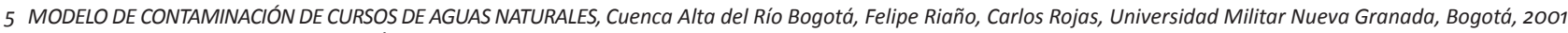

6 DIAGNOSTICO AMBIENTAL Y LEGALIZACIÓN DE LAS INDUSTRIAS DEL CUERO, CAR - Cundinamarca, Bogotá, Diciembre de 1999. 


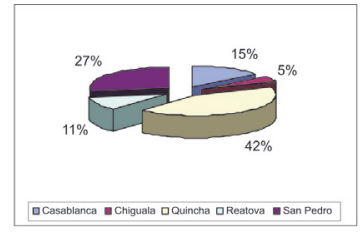

\begin{tabular}{|l|r|}
\hline \multicolumn{1}{|c|}{ VEREDA } & N $^{\mathbf{0}}$ INDUSTRIAS \\
\hline Casablanca & 19 \\
\hline Chiguala & 6 \\
\hline Quincha & 56 \\
\hline Reatova & 14 \\
\hline San Pedro & 36 \\
\hline TOTAL & $\mathbf{1 3 1}$ \\
\hline
\end{tabular}

Figura 3. Distribución de industrias por vereda Fuente: CAR-Cundinamarca, 1992

En la Figura 4 se muestra la localización del sector de San Benito y su proximidad al río Tunjuelito, afluente del río Bogotá, en el cual se producen los vertimientos de este sector.

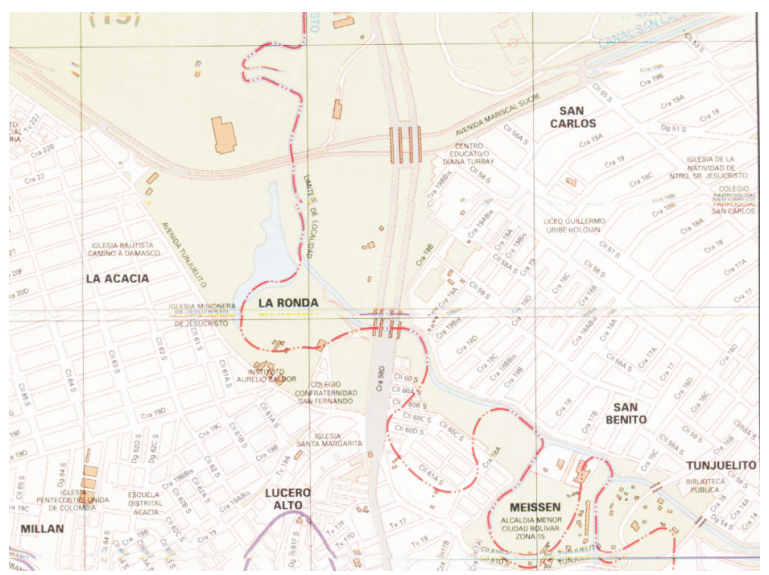

Figura 4 Sector de San Benito.

FUENTE: Santafé de Bogotá Calle por Calle, Plano 65, Las AmariIlas de Combiser, 2000

\section{LOS PROCESOS EN LAS CURTIEMBRES}

En el proceso industrial de la producción de cuero, se hace generalmente como se muestra la Figura $5^{7}$, siendo el agua el elemento fundamental en estos procesos.

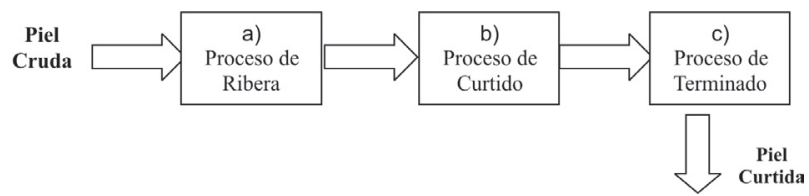

Figura 5. Fases del proceso general de producción de cuero Fuente: Corporación Autónoma Regional de Cundinamarca, 2000
Proceso de ribera: llamado también de limpieza ${ }^{8}$, en esta fase se hace la recepción de la materia prima y se elimina la grasa, carne y pelo, hasta el dividido de pieles. En la planta de sacrificio y faenado, las reses son desolladas dejando caer las pieles "al piso" en donde se mezclan con otros residuos propios de la preparación de la carne en canal para su distribución a los centros de consumo. Es así, como al llegar la piel al sitio de tratamiento se encuentra con residuos de tierra, sangre, estiércol, grasa, pelo y otras sustancias, además de la gran cantidad de sal que se aplica

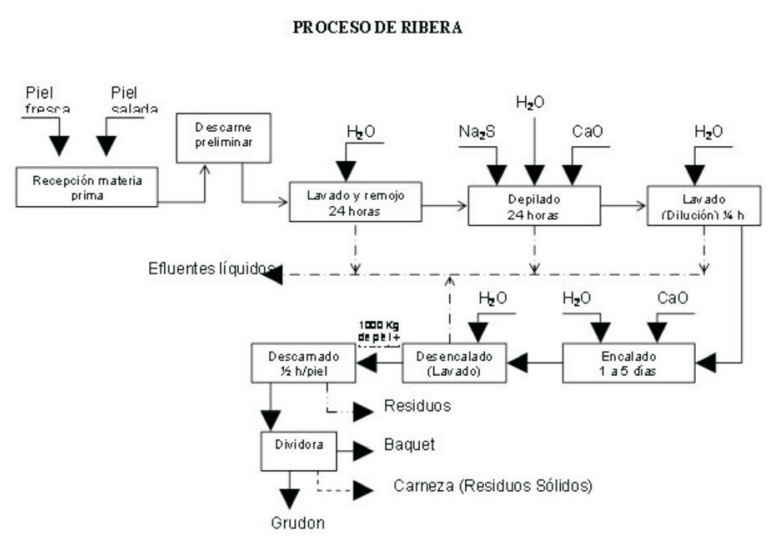

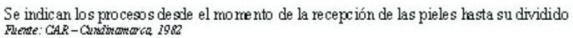

Figura 6. El proceso de ribera

Fuente: CAR-Cundinamarca, 2000

Estas sustancias son las primeras que se remueven en la curtiembre, algunas en forma manual y otras colocando las pieles en fulones (Figura 7) con agua y sometiéndolas a "lavado" (mezclado) con adición de sulfuro de sodio, cal humectante y óxido de calcio, además de las que son propias de este proceso, como se indica en la Figura 6. Los residuos líquidos que se obtienen presentan altos niveles de alcalinidad, sulfuro, nitrógeno, sólidos disueltos y suspendidos, aceite y grasa y altas demandas de DBO5 y DQO. 


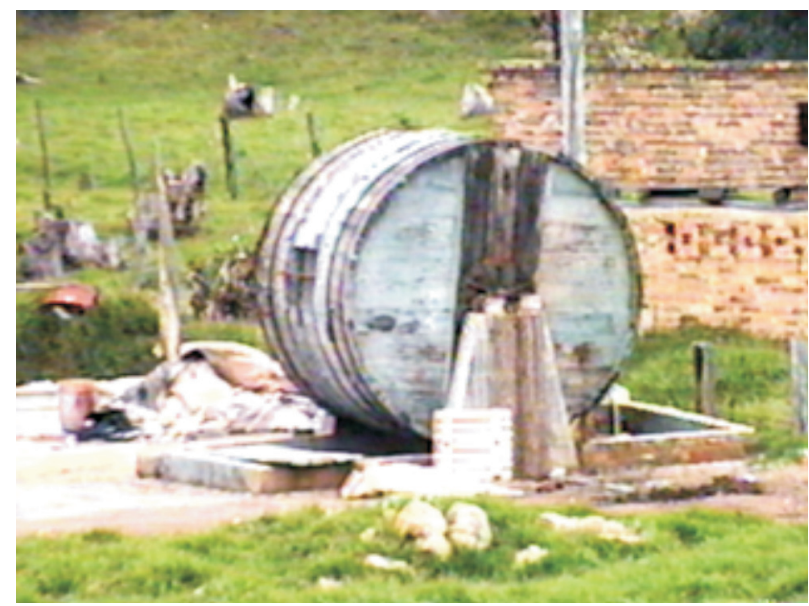

Figura 7. Operación de mezclado en el tratamiento de pieles Cortesía de Ings. Felipe Riaño y Carlos Rojas

En esta fase se produce la mayor cantidad de residuos sólidos en forma de carne, polvo arenoso, recortes de las pieles, lodos grasas y otros. Algunos pueden ser reciclados como el pelo y las grasas.

El proceso de curtido: tiene por objeto producir un material resistente a los efectos de la descomposición de tipo físico o biológico, se puede realizar de dos formas diferentes según el producto que se espere obtener, en la siguiente manera:

Proceso de curtido con cromo: se utiliza para elaborar cueros de textura suave y calibre delgado para aplicación en el calzado y marroquinería, mediante la aplicación de cromo, sales metálicas y formaldehídos, con el fin de ablandarlas y manejarlas para obtener el producto final. Se produce efluentes líquidos baja demanda de $\mathrm{DBO} 5$ y DQO y altos contenidos de sales y ácidos, y además algunos residuos sólidos como residuos de pelo, bordes, carne y grasa. En el Figura 8, se muestra la secuencia de este proceso.

Proceso de curtido con tanino: el curtido vegetal o con tanino produce un cuero grueso resistente y poco flexible, utilizado en la industria del calzado. En este proceso se agregan al agua: bisulfato de sodio, sulfato de amonio y enzimas, se aprovecha las propiedades curtientes de ciertos vegetales como el castaño, el quebracho y la mimosa, con gran exigencia de oxígeno. Estos materiales se obtienen a partir de cortezas de árboles que poseen alto contenido de componentes tánicos orgánicos. Se producen amoniaco, desechos alcalinos, ácidos y agua altamente coloreada como se indica en la Figura 9.

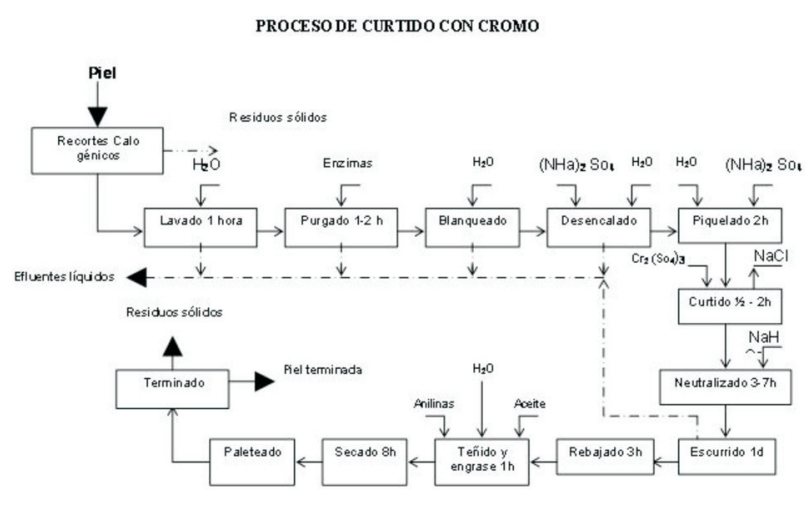

Se utiliza en la elaboración de cueros para namoquinería

Figura 8. El proceso de curtido con cromo

Fuente: CAR-Cundinamarca

PROCESO DE CURTIDO CON TANINO

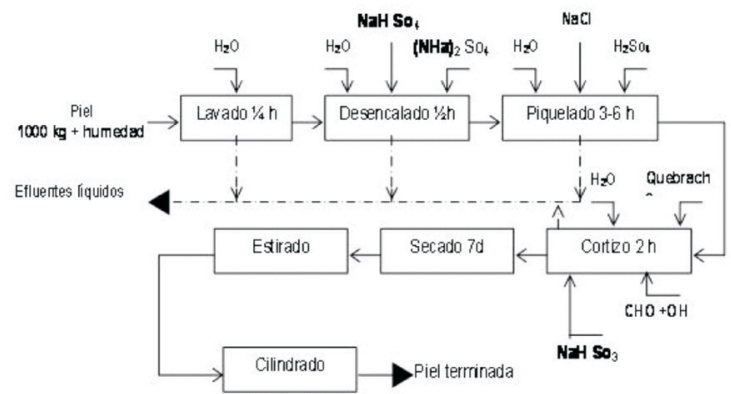

Se utiliza par a elaborar cueros gruesos resistertes y poco flexibles en la industria del calzado Finente: CAR - Cold numurea, 1982

Figura 9. El proceso de curtido con tanino Fuente CAR-Cundinamarca 
En una última etapa de estos procesos llamada recurtido, se realiza el teñido mediante la adición de tintas y aceites para reemplazar los aceites naturales de las pieles (licor grasoso), se generan efluentes concentrados que contienen aceite y color. Los tres procesos mencionados anteriormente, se realizan en el mismo fulón (tambor).

Proceso de terminado: una vez ejecutados los procesos anteriores, las pieles se tiñen y engrasan, se secan, se sujetan con estacas u otro soporte para mantenerlas estiradas y se lavan para su entrega al consumidor. Se presentan altas descargas de colorantes y grasas.

El manejo inadecuado de las curtiembres puede llevar a la adquisición de la dermatitis y el ántrax, causada por el contacto con los químicos y las pieles, y otras enfermedades de tipo respiratorio por la exposición a los polvos y las emisiones gaseosas de las sustancias químicas empleadas.

\section{ASPECTOS FíSICO-QUÍMICOS}

En la industria de las curtiembres se manejan residuos sólidos, líquidos y gaseosos. Los residuos sólidos se producen tanto en la curtición como en los productos terminados, aunque estos no son de interés para este análisis, tampoco se desconoce su influencia en la caracterización de los residuos líquidos producidos. Se consideran de mayor interés: la sal utilizada en la conservación de las pieles hasta su tratamiento, el pelo, los descarnes (crudos y apelambrados) y los lodos. Algunos gases están relacionados con los procesos químicos que se utilizan en esta industria. Los residuos líquidos son el objetivo de este análisis, por tanto se enfatiza en ellos con especial dedicación a los procesos de curtición

Cuando se analizan los residuos líquidos de las curtiembres, diferentes autores manejan con diferentes conceptos cuales son las variables que se deben con- siderar con mayor interés. En este caso, atendiendo a los escritos que se han consultado, se eligieron como variables más representativas, entendiendo como tales aquellas que están involucradas con los mayores problemas de contaminación, las siguientes: $\mathrm{pH}$, Temperatura del agua, Aceites y grasas, Conductividad, Cloruros, DBO5, DQO, Nitrógeno total, Sólidos suspendidos totales (SST), Sulfuros y Cromo total, este último es el mayor incidencia contaminante producida por la industria de las curtiembres.

Orientativamente, en la Tabla 1 se resumen los valores para las variables indicadas de acuerdo con los registros de la CAR - Cundinamarca en 1982, cuya representación gráfica se muestra en la Figura 10.

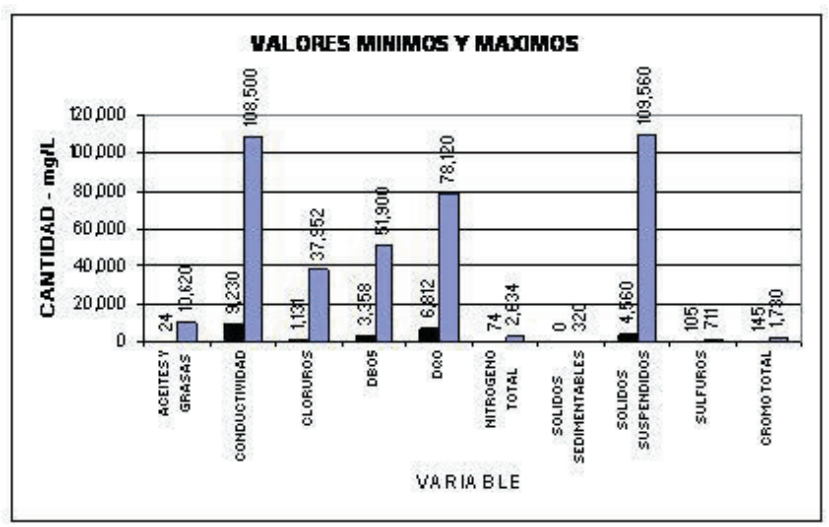

Figura 10. Valores Máximos Y Mínimos

Fuente: CAR - Cundinamarca, 1982

Tabla 1. Valores Máximos Y Mínimos

\begin{tabular}{|l|c|r|r|}
\hline \multicolumn{1}{|c|}{ VARIABLE } & UNIDAD & MÍNIMO & MÁXIMO \\
\hline ACEITES Y GRASAS & $\mathrm{mg} / \mathrm{L}$ & 24 & 10,620 \\
\hline CONDUCTIVIDAD & $\mathrm{uS} / \mathrm{cm}$ & 9,230 & 108,500 \\
\hline CLORUROS & $\mathrm{mg} / \mathrm{L}$ & 1,131 & 37,952 \\
\hline DBO $_{5}$ & $\mathrm{mg} / \mathrm{L}$ & 3,358 & 51,900 \\
\hline DQO & $\mathrm{mg} / \mathrm{L}$ & 6,812 & 78,120 \\
\hline NITRÓGENO TOTAL & $\mathrm{mg} / \mathrm{L}$ & 74 & 2,634 \\
\hline $\begin{array}{l}\text { SÓLIDOS } \\
\text { SEDIMENTABLES }\end{array}$ & $\mathrm{mg} / \mathrm{L}$ & 0 & 320 \\
\hline SÓLIDOS SUSPENDIDOS & $\mathrm{mg} / \mathrm{L}$ & 4,560 & 109,560 \\
\hline SULFUROS & $\mathrm{mg} / \mathrm{L}$ & 105 & 711 \\
\hline CROMO TOTAL & $\mathrm{mg} / \mathrm{L}$ & 145 & 1,730 \\
\hline
\end{tabular}

Vol. 16 - No. 2 • Diciembre 2006 
En diciembre de 1999, Unidad Especial de Curtiembres de la CAR-Cundinamarca, entrega el documento "Diagnóstico ambiental y legalización de las industrias del cuero", en el que se presenta la actualización del inventario de curtiembres registradas en su jurisdicción, se informa sobre los impactos ambientales y con base en una evaluación tecnológica se plantea la metodología y procedimientos con los cuales deben cumplir las personas y empresas dedicadas a la industria de las curtiembres, sin embargo, no se hace ningún reporte de los valores de las variables relacionadas con los vertimientos que estas realizan.

En junio de 2001, el IDEAM publica el "Diagnóstico ambiental y lineamientos para el uso del área de Villapinzón - Chocontá, (Cundinamarca)", en el cual se realiza una evaluación general de la situación ambiental del área mencionada en la que se incluyen las curtiembres.

De los estudios realizados, manifiestan que para los análisis de la información se presentan varias dificultades (Ibid, pág 191), se transcriben entre ellas:

- "Se comparan muestras de líquidos concentrados (fulones y muestras de piso) con muestras de aguas residuales (vertimientos),

- En los muestreos no se reporta el caudal del vertimiento o el volumen del fulón o tanque,

- No se conoce la metodología y procedimiento en la recolección de muestras (si fue simple o compuesta),

- No se consigna cual es período de funcionamiento (año, mes, día), dato que es importante dada la discontinuidad de los vertimientos,

- No todas la unidades tienen datos de vertimientos, ni cantidad de pieles procesadas al mes"

Se incluyen en este informe los datos reportados por en IDEAM en las Tablas 2 a 4, obtenidos con base en estudios de caso usando factores de consumo y de salidas por unidad de producto (Ibid, pág 186), es decir, por tonelada de piel final curtida, atendiendo a las observaciones anteriormente anotadas. Adicionalmente, se encontró una alta dispersión en los valores de las variables físico-químicas que afectan la calidad de la información, por tanto, tales datos se deben observar en forma conservadora.

Tabla 2. Variables físico-químicas sobre 104 expedientes

Fuente: Diagnóstico ambiental y lineamientos para el uso sostenible del área de Villapinzón - Chocontá, Cundinamarca, IDEAM, 2001

\begin{tabular}{||l|c|r|r|r|}
\hline \multicolumn{1}{|c|}{ VARIABLE } & UNIDAD & PROMEDIO & \multicolumn{1}{c|}{ MAXIMO } & \multicolumn{1}{c|}{ MINIMO } \\
\hline ACEITES Y GRASAS & $\mathrm{mg} / \mathrm{L}$ & 1473,1 & 21345 & 0,0 \\
\hline CONDUCTIVIDAD & $\mathrm{S} / \mathrm{cm}$ & 33407,9 & 158200 & 524,0 \\
\hline CLORUROS & $\mathrm{mg} / \mathrm{L}$ & 9235,3 & 78587 & 185,0 \\
\hline DBO ${ }_{5}$ & $\mathrm{mg} / \mathrm{L}$ & 9834,6 & 83650 & 15,0 \\
\hline DQO & $\mathrm{mg} / \mathrm{L}$ & 20019,0 & 322207 & 67,3 \\
\hline NITROGENO TOTAL & $\mathrm{mg} / \mathrm{L}$ & 973,7 & 14606 & 0,2 \\
\hline SOLIDOS SEDIMENTABLES & $\mathrm{mg} / \mathrm{L}$ & 55,7 & 3050 & 0,0 \\
\hline SOLIDOS SUSPENDIDOS & $\mathrm{mg} / \mathrm{L}$ & 13832,1 & 103900 & 45,0 \\
\hline SULFUROS & $\mathrm{mg} / \mathrm{L}$ & 355,3 & 7845 & 0,0 \\
\hline CROMO TOTAL & $\mathrm{mg} / \mathrm{L}$ & 3915,8 & 72180 & 0,2 \\
\hline TEMPERATURA & ${ }^{\circ} \mathrm{C}$ & 15,0 & 21 & 11,0 \\
\hline pH & & 11,5 & 13,9 & 3,2 \\
\hline E-Coli & $\mathrm{NMP} / 100 \mathrm{ml}$ & 270,0 & 24000 & 0,0 \\
\hline
\end{tabular}
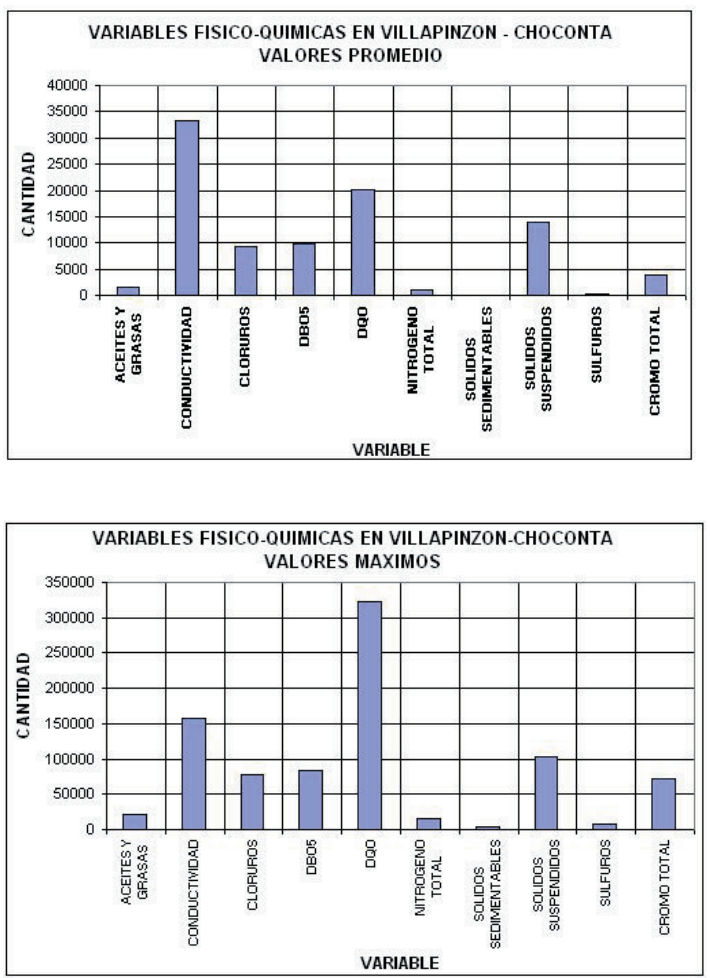
UNIVERSIDAD MILITAR NUEVA GRANADA

Tabla 3. Variables físico químicas de las aguas residuales de las curtiembres en villapinzón

Fuente: Diagnóstico ambiental y lineamientos para el uso sostenible del área de Villapinzón - Chocontá, Cundinamarca, IDEAM, 2001

\begin{tabular}{|c|c|c|c|c|c|c|c|}
\hline \multicolumn{8}{|c|}{ 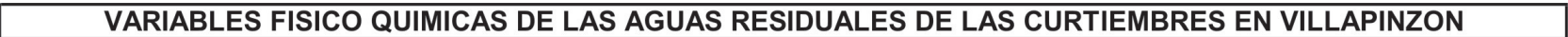 } \\
\hline VARIABLE & UNIDAD & PROCE & SO CON TA & NINO & PROCE & SO CON CF & DMO \\
\hline & & PROMEDIO & MAXIMO & MINIMO & PROMEDIO & MAXIMO & MINIMO \\
\hline $\mathrm{DBO}_{5}$ & $\mathrm{mg} / \mathrm{L}$ & $4.777,0$ & $8.970,0$ & $1.497,0$ & 912,0 & $1.350,0$ & 595,0 \\
\hline DQO & $\mathrm{mg} / \mathrm{L}$ & $23.715,0$ & $46.151,0$ & $3.773,0$ & $9.100,0$ & $9.922,0$ & $3.334,0$ \\
\hline NITROGENO AMONIACAL & $\mathrm{mg} / \mathrm{L}$ & 34,1 & 53,1 & 22,3 & 59,5 & 88,0 & 36,1 \\
\hline SOLIDOS TOTALES & $\mathrm{mg} / \mathrm{L}$ & $7.572,0$ & $10.756,0$ & $5.000,0$ & $5.425,0$ & $8.329,0$ & $3.250,0$ \\
\hline SOLIDOS SUSPENDIDOS TOTALES & $\mathrm{mg} / \mathrm{L}$ & $1.550,0$ & $2.184,0$ & $1.180,0$ & $1.129,0$ & $1.845,0$ & 561,0 \\
\hline SOLIDOS SUSPENDIDOS VOLATILES & $\mathrm{mg} / \mathrm{L}$ & 928,0 & $1.283,0$ & 640,0 & 663,0 & $1.070,0$ & 359,0 \\
\hline $\mathrm{pH}$ & & 8,9 & 10,2 & 8,0 & 10,4 & 12,2 & 9,0 \\
\hline TEMPERATURA & ${ }^{\circ} \mathrm{C}$ & 17,5 & 18,9 & 16,5 & 17,9 & 18,7 & 17,3 \\
\hline
\end{tabular}
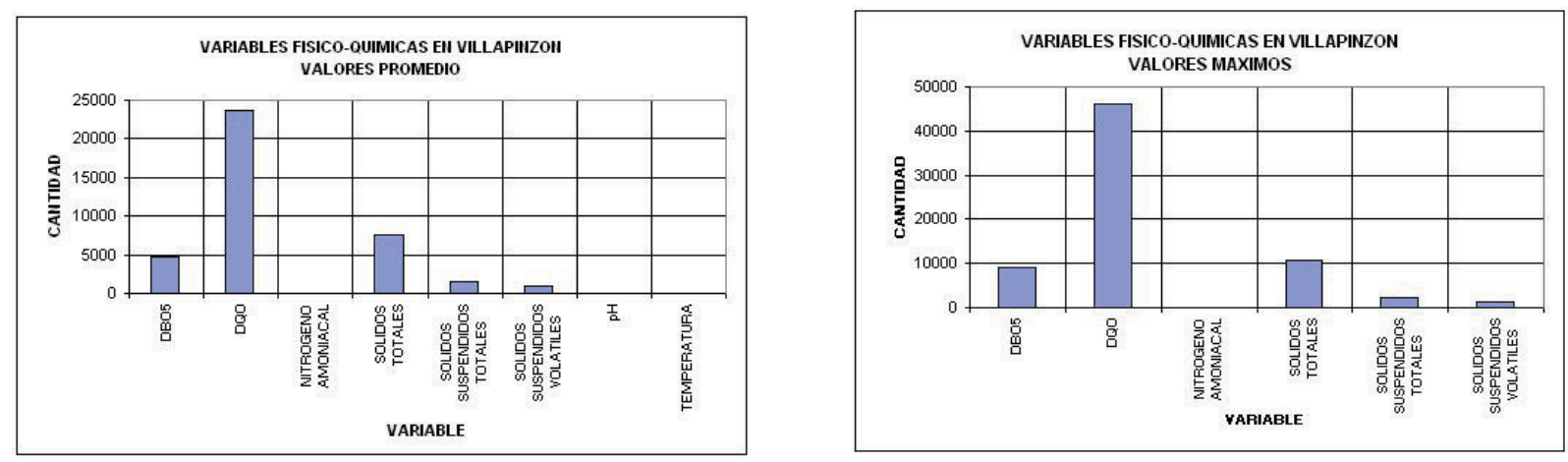

Tabla 4. Valores de carga estimados para limites de producción

Fuente: Diagnóstico ambiental y lineamientos para el uso sostenible del área de Villapinzón - Chocontá, Cundinamarca, IDEAM, 2001

\begin{tabular}{|c|c|c|c|c|c|c|c|c|c|c|}
\hline \multicolumn{11}{|c|}{ VALORES DE CARGA ESTIMADOS PARA LIMITES DE PRODUCCION } \\
\hline \multirow[t]{2}{*}{ VARIABLE } & UNIDAD & \multicolumn{3}{|c|}{ PRODUCCION MAXIMA } & \multicolumn{3}{|c|}{ PRODUCCION MEDIA } & \multicolumn{3}{|c|}{ PRODUCCION MINIMA } \\
\hline & CONCEN & PROMEDIO & MAXIMO & MINIMO & \begin{tabular}{|l|} 
PROMEDIO \\
\end{tabular} & MAXIMO & MINIMO & PROMEDIO & MAXIMO & MINIMO \\
\hline $\mathrm{DBO}_{5}$ & $\mathrm{mg} / \mathrm{L}$ & 55,1 & 81,51 & 35,9 & 35,85 & 53,7 & 23,69 & 16,63 & 24,62 & 10,85 \\
\hline DQO & $\mathrm{mg} / \mathrm{L}$ & 549,9 & 599,06 & 201,3 & 357,7 & 390,01 & 131,05 & 165,97 & 180,96 & 60,81 \\
\hline SOLIDOS TOTALES & $\mathrm{mg} / \mathrm{L}$ & 327,6 & 502,88 & 196,2 & 213,25 & 327,4 & 127,75 & 98,94 & 151,91 & 59,28 \\
\hline SOLIDOS SUSPENDIDOS TOTALES & $\mathrm{mg} / \mathrm{L}$ & 68,2 & 111,4 & 33,9 & 44,38 & 72,52 & 22,05 & 20,59 & 33,65 & 10,23 \\
\hline SOLIDOS SUSPENDIDOS VOLATILES & $\mathrm{mg} / \mathrm{L}$ & 40,0 & 64,6 & 21,7 & 26,06 & 42,06 & 14,11 & 12,09 & 19,52 & 6,55 \\
\hline NITROGENO AMONIACAL & $\mathrm{mg} / \mathrm{L}$ & 3,6 & 5,31 & 2,2 & 2,34 & 3,46 & 1,42 & 1,09 & 1,61 & 0,66 \\
\hline $\mathrm{pH}$ & & 10,4 & 12,2 & 9,0 & 10,4 & 12,2 & 9 & 10,4 & 12,2 & 9 \\
\hline
\end{tabular}
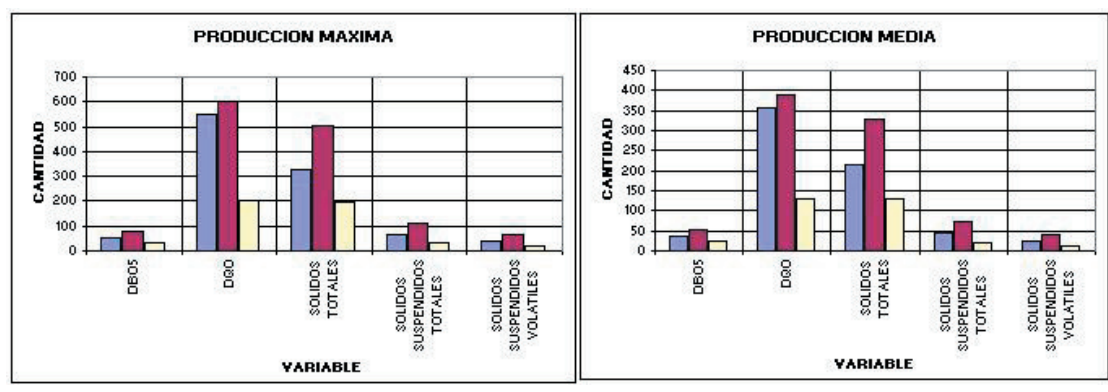

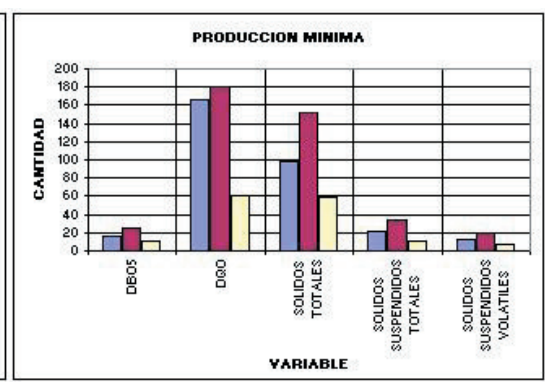


En general, se observa que los procesos artesanales tienen los mayores consumos de insumos, en especial el cromo, sin embargo, los sulfuros presentan un consumo menor, teniendo en cuenta que para las industrias medianas y grandes los cueros ya han sido pelados en procesos diferentes. En el consumo de insumos químicos las industrias medianas representan un $80 \%$ del consumo total, las grandes un $9 \%$ y las pequeñas un $11 \%$, y proporcionalmente es su generación de cargas contaminantes ${ }^{9}$.

En Bogotá la industria de las curtiembres se encuentran centralizadas en el sector de San Benito. Sobre este sector no fue posible obtener información cuantitativa, actualmente se encuentran trabajando como industrias independientes (300) pero algunas de ellas se han asociado en una cooperativa (Copicur). Existe un plan a nivel local para desarrollar un proyecto conocido como ECOPARQUE SAN BENITO, en el cual se aplicara la técnica del WET-BLUE para la remoción parcial del cromo y la construcción de una planta de tratamiento de los efluentes de cada industria, los cuales serian conducidos a esta planta, mediante el alcantarillado sanitario en donde se mezclan con las aguas residuales domésticas del sector, sin embargo los operadores y dueños de las curtiembres manifiestan su interés por construir un sistema de alcantarillado de reúna las aguas residuales de cada industria y las conduzca a la planta de tratamiento.

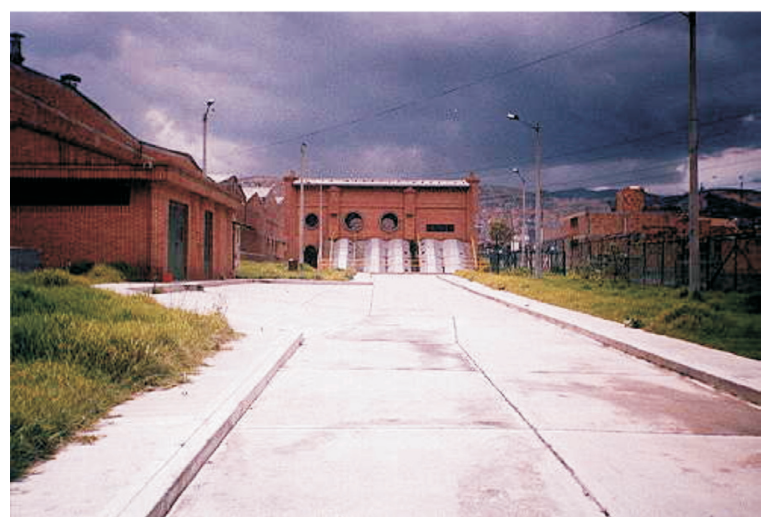

Figura 11. Vista General de la planta de tratamiento de San Benito en Bogotá

9 IDEAM, Junio de 2001
La técnica del WET-BLUE consiste fundamentalmente en precipitar el cromo elevando el $\mathrm{pH}$ y separarlo del flujo en un tanque, para procurar su reuso. El sistema de recuperación del cromo consiste en el empleo de un depósito de recepción, un sistema de homogenización, tanques de precipitación y reacción, tanques de almacenamiento y empaque. De esta forma, en los vertimientos de aguas residuales solamente iría el cromo residual que pueda quedar suspendido en el efluente.

\section{ANÁLISIS DE LOS RESIDUOS}

La descripción resumida de los procesos requeridos para la industria de las curtiembres es la que se indicó anteriormente, con base en los cuales se busca caracterizar los residuos líquidos producidos. Es así como diversos autores sugieren la tendencia dominante sobre aquellos que pueden ser considerados de mayor interés como efluentes contaminantes, algunos de ellos reportan valores cuantitativos sobre las variables que son de interés particular.

Para este análisis se adoptó como criterio utilizar como marco de referencia para juzgar su influencia en los recursos hídricos superficiales, el Decreto 475 sobre los requisitos calidad de agua potable, el Decreto 1594 sobre calidad de agua para vertimiento a nivel nacional y algunos valores dados en la Resolución 55 del DAMA-Bogotá, también sobre calidad de vertimientos en el distrito capital.

Atendiendo a la información disponible, se anotan las siguientes observaciones:

- Considerando las variables que convencionalmente son susceptibles de ser medidas con fines de análisis de calidad de agua para diferentes usos como se indico anteriormente, se aprecia que en el caso de las curtiembres las diferentes entidades analizan algunas que comunes pero con diferentes 
modalidades, por ejemplo, en el caso de los sólidos se realizan diferentes ensayos según el interés particular de cada entidad.

- Sierra (1997), recomienda la medición de conductividad, sólidos suspendidos totales y temperatura del agua en el contexto de los aspectos físicos, en los cuales se mantiene concordancia con los analizados por las entidades. Teniendo en cuenta que los sólidos también representan parcialmente la materia orgánica y que de su concentración dependerá en buena parte el diseño de ciertas estructuras y selección de equipos, estas variables deben ser medidas en forma integral.

- En cuanto hace referencia a los aspectos químicos se recomienda la medición de alcalinidad, cromo, grasas y aceites, nitrógeno, pH, DQO y DBO5, especialmente (Sierra, 1997), con la misma observación anterior. En este sentido es más fácil la selección de las variables a medir para los residuos de las curtiembres, dada la experiencia que existe al respecto sobre aquellos contaminantes que cabe esperar se produzcan como residuo, en función del conocimiento previo que hay de los insumos utilizados para el tratamiento de las pieles.

- Desde el punto de vista biológico, no existe realmente un seguimiento y menos normas decididas sobre ellos.

- Curiosamente, en los reportes analizados no se aprecia interés por medición de la magnitud de los caudales efluentes de la industria de las curtiembres. Queda entonces la incertidumbre sobre las concentraciones con las que se vierten los contaminantes a los cursos de agua superficiales, solamente se advierte de su presencia y la magnitud "absoluta" de su medida.

- Las mediciones obedecen a muestreos puntuales en el tiempo y en el espacio, posiblemente sufi- cientes para un control de tipo industrial tendiente a establecer los requerimientos exigidos para el cumplimiento de las normas legales a que están sometidas estas industrias, por tanto, se carece de un conocimiento cierto sobre la influencia que ejercen estos contaminantes sobre los cuerpos de agua en los cuales se vierte el efluente contaminado, especialmente dependiendo de la época del año.

- La magnitud de las variables presenta amplios rangos de oscilación, dificultando la determinación de sus valores característicos en el efluente. Orozco (1987) presenta un estimativo sobre algunas de estas variables para los efluentes de las curtiembres en la ciudad de Medellín.

- Resulta difícil recomendar la obtención de valores promedio de los contaminantes presentes en las aguas residuales de las curtiembres debido a diferentes factores: Las variables de tipo químico son susceptibles al entorno en el cual se analizan, las condiciones de captación y transporte de muestras (que deben realizarse en forma cuidadosa), los ensayos también dependen de la subjetividad del laboratorista con relación a su experiencia y detalle con el que se realicen las pruebas y finalmente las afectaciones en la misma curtiembre desde el momento en que el efluente abandona el fulón. Existe también gran dependencia de la variación en la producción, en función del movimiento del mercado de las curtiembres procesadas y de la disposición de las pieles "crudas".

- El cromo siempre muestra variaciones altamente significativas en función de la fecha pero independientemente del sitio en el cual se halle localizada geográficamente, como se observa en la Tabla 4.

- La temperatura muestra un valor relativamente estable alrededor de los $20^{\circ} \mathrm{C}$. 
- El proceso con mayor producción de residuos es el de ribera, seguido de los de curtido y disminuye en el proceso de terminado.

En general, los efluentes de las curtiembres en los registros observados presentan variaciones significativas entre los valores mínimos y máximos como se observa en las tablas antes mencionadas, los cuales siempre superan ampliamente los límites establecidos por la norma legal.

\section{EFECTOS SOBRE EL MEDIO AMBIENTE}

A nivel mundial se reconoce esta industria como altamente contaminante produciendo degradación ambiental muchas veces de carácter irreversible, independientemente del tamaño de la industria teniendo en cuenta que sus impactos cualitativos y cuantitativos sobre las componentes físico-biótica y socioeconómica.

Tabla 5. Curtido con cromo municipio de Villapinzón
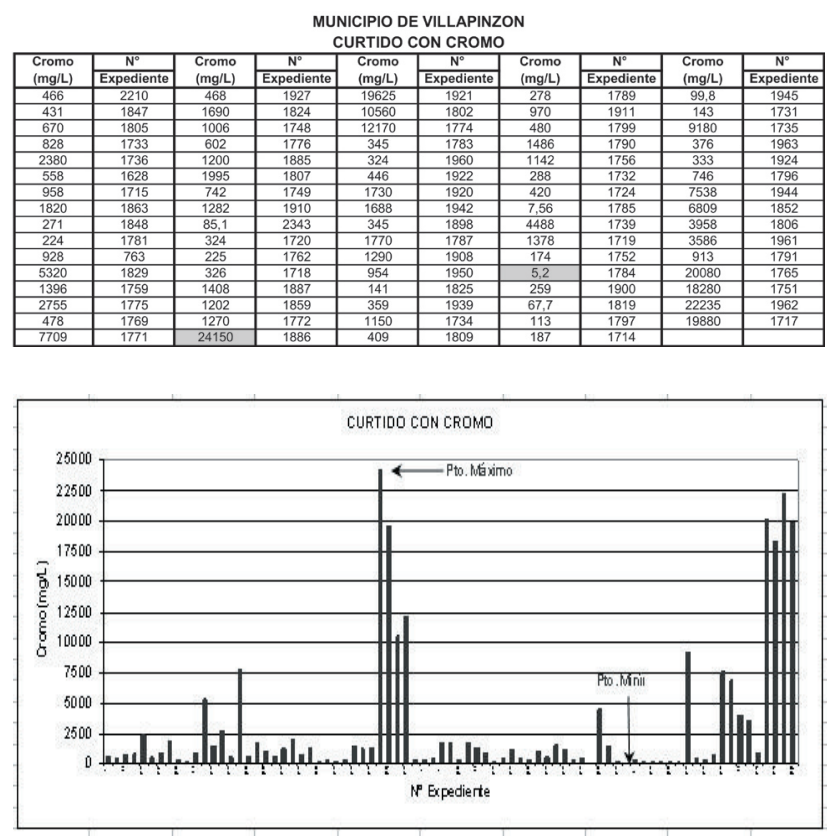

En la componente física los residuos de las curtiembres causan sobre el agua efectos negativos diminuyendo la presencia de oxígeno disuelto, aumenta la salinidad y otros efectos, es más grave sobre las aguas subterráneas debido a la falta de aireación. En el suelo altera su textura y estructura, especialmente en relación con su porosidad, con la consecuente aceleración de la erosión, disminución de la productividad agrícola y pérdida de la permeabilidad. Al ocupar los residuos líquidos los vacíos del suelo, especialmente las grasas y aceites, se altera la aireación y el flujo del agua en el medio poroso alterando las condiciones de drenaje, se transportan entonces en forma lenta los contaminantes a las zonas de recarga tanto de aguas superficiales como subterráneas. La calidad del aire se afecta especialmente por la producción de olores y gases, causados por la descomposición de la materia orgánica y la reacción de algunos agentes químicos utilizados en las curtiembres con el aire, tal es el caso de los sulfuros que pueden generar la producción de gas sulfhídrico el cual en pequeñas cantidades se identifica por su mal olor, pero en concentraciones mayores es tóxico, el amoniaco utilizado también es generador de malos olores.

Sobre la componente biótica es innegable el efecto negativo sobre la vida acuática, destruye casi y completamente la microbiota que constituye la base de la vida de algunas especies superiores como micro y macroinvertebrados y por ende las especies de peces así mismo se genera el desplazamiento de anfibios y especies mayores que disponen de estas fuentes como abrevaderos. Adicionalmente se rompe la cadena de procesos de autodepuración natural de las corrientes de agua debido a la disminución del oxígeno disuelto y la afectación sobre las especies vegetales vecinas al cauce de las corrientes superficiales receptoras, algunas de las cuales no están capacitadas para el manejo de los contaminantes presentes en el agua, entre otros. 
En la componente socioeconómica se observa un efecto positivo que es la generación de empleo, en particular en Colombia esta industria todavía tiene altas aplicaciones de tipo artesanal realizada por campesinos y personas de bajos recursos y para quienes puede llegar a ser su medio de subsistencia. Los principales efectos negativos corresponden a la elevada dificultad para utilizar las aguas contaminadas por estos residuos en el abastecimiento de agua potable, dados los altos costos que implica su tratamiento. Otro efecto de alta relevancia es su influencia sobre la salud tanto en el proceso productivo debido al manejo de los insumos utilizados sin las debidas normas de seguridad industrial como por los productos y subproductos entregados al medio ambiente. Tienen especial interés las enfermedades de la piel (dermatitis), sistema respiratorio (inhalación de gases) y sistema digestivo (úlceras).

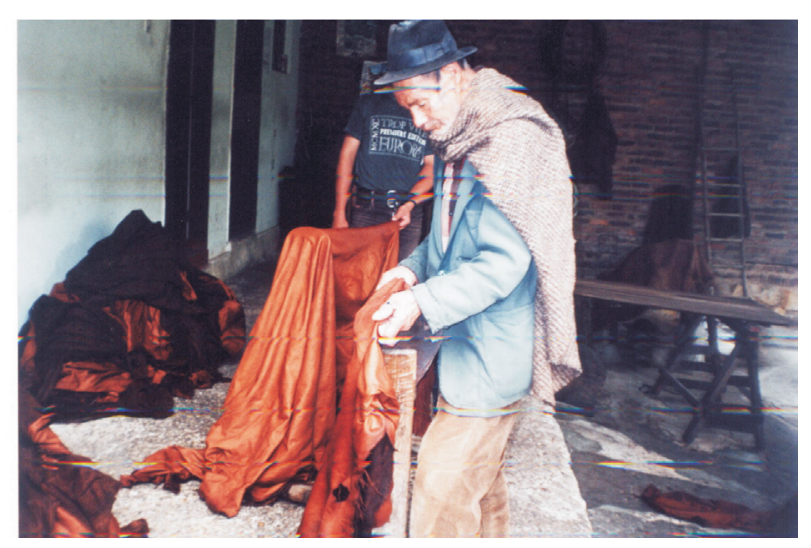

Figura 12. Manejo artesanal de las curtiembres Fuente: IDEAM

En los estudios ambientales también se reconoce el entorno legal y normativo, pues mediante estos se regulan las condiciones de uso de los recursos naturales, con base en esta reglamentación se fijan los planes y programas de desarrollo de la comunidad para mantener un medio ambiente sostenible y sustentable.

\section{MANEJO DE LOS RESIDUOS LIQUIDOS}

Existen diversas tendencias en el mercado para optimizar la industria de las curtiembres tanto en el ámbito tecnológico como de oferta ambiental.

En este sentido, se considera particularmente el tratamiento del cromo y su recuperación durante el proceso de curtición. Una de las técnicas que esta adquiriendo buena difusión, es la conocida como WET-BLUE, la cual se aplica a industrias suficientemente grandes con capacidad para soportar con beneficio los costos que implica su empleo. En nuestro medio apenas existen planes de utilización de esta tecnología como es el caso del Ecoparque San Benito en Bogotá.

Para las características del entorno y de las personas que se dedican en Colombia a la industria de las curtiembres, se plantean dos modalidades de solución para el manejo de residuos líquidos de esta industria: una para los pequeños productores y otra para los medianos y grandes productores. En el primer caso parece razonable el empleo de humedales artificiales y en el segundo el sistema tradicional de lodos activados (en revisión).

La técnica de los humedales artificiales consiste en el empleo de un lecho filtrante sobre el que se dispone una lámina de agua. Sobre el lecho filtrante se colocan plantas de especies tales que puedan adherir a su raíz parte de las sustancias contaminantes (alisos y cañas), otra parte de ellas es retenida por el medio filtrante y los residuos que pasan al efluente deben cumplir con las características de vertimiento exigidas. Para lograr un funcionamiento satisfactorio se recomienda que se realice una limpieza semanal, con el fin de evitar su colmatación y propagación de olores ${ }^{10}$. 

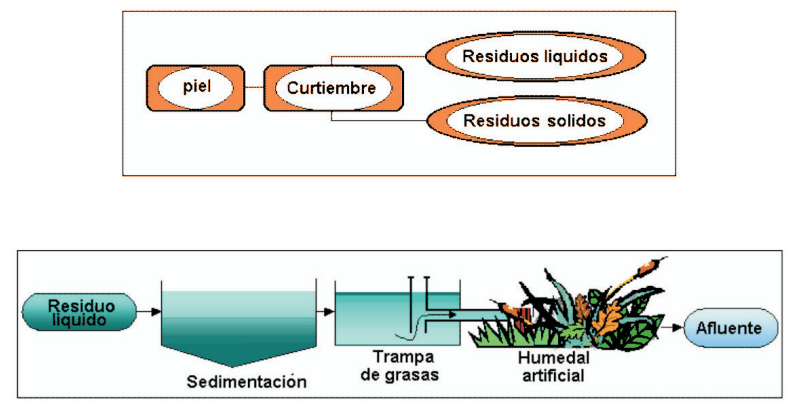

Figura 13. Solución con humedal artificial

En la Figura 13 se incluye un esquema del humedal artificial y de la solución propuesta para el manejo de los residuos líquidos de las curtiembres, la cual consiste en disponer de un tanque de recepción de las aguas residuales en el cual se produce una sedimentación del material granular seguida de una trampa de grasas para conducir las aguas al humedal artificial y finalmente realizar la entrega del efluente al receptor final.

Para el caso de los medianos y grandes productores se propone como alternativa de solución la técnica convencional de lodos activados (en revisión). En la Figura 14 se muestra un esquema de la solución propuesta.

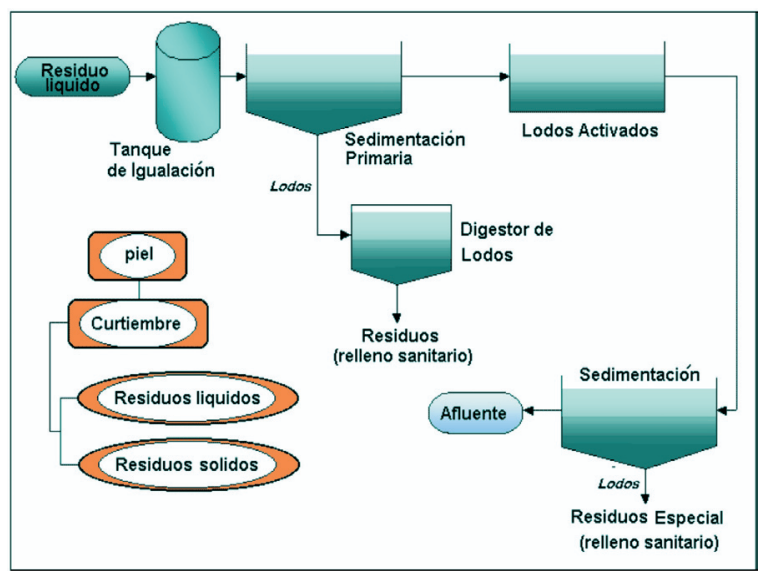

Figura 14. Solución con lodos activados
Esta solución consiste en la utilización de un tanque de igualación como receptor del residuo líquido, teniendo en cuenta la intermitencia del flujo, seguido de un tanque sedimentador en donde se realiza una sedimentación primaria con la finalidad de retener el material granular que pueda estar presente en el agua y el aislamiento de las grasas y aceites; en este se produce la retención de los lodos para su disposición en un relleno sanitario especial y de ser posible su utilización para fabricación de compostaje. Enseguida se utiliza la técnica de lodos activados en especial la modalidad con flujo a pistón, flujo continuo y mezcla completa ${ }^{11}$ con la finalidad de su aplicabilidad a los grandes productores de curtiembres y a las altas concentraciones de contaminantes presentes en el agua residual. Inmediatamente después se produce la sedimentación de los lodos resultantes para su disposición en un relleno sanitario especial y el vertimiento del efluente a su disposición final.

Cabe anotar, que en el mercado existen técnicas altamente sofisticadas algunas de ellas en vía de experimentación para el tratamiento específico de las aguas residuales provenientes de la industria de las curtiembres. tal es el caso del Proyecto LIFE auspiciado por IPPC, según el cual se instaló en septiembre de 2002, la planta Curtidos Mare Nostrum, atendiendo a las directivas del Instituto Tecnológico del Calzado y Conexas (INESCOP), quien se encarga de su operación y observación ${ }^{12}$.

\section{CONCLUSIONES}

Actualmente, se están utilizando materiales de tipo sintético (polímeros) en algunos de los procesos del curtido con la finalidad de disminuir la contaminación producida por esta industria.

La medición de los efluentes en las plantas de procesamiento de pieles en Colombia presenta gran re-

11 TRATAMIENTO DE AGUAS RESIDUALES EN POBLACIONES PEQUEÑAS, Crites, Tchobanoglous, Ed. McGraw-Hill, Bogotá, 2000

12 RECIRCULACION DE BAÑOS DE CURTICION EN LAS INDUSTRIAS DE CURTIDOS, INESCOP, 2002 
sistencia por parte de sus propietarios, pues debido a las actuales exigencias ambientales de obligatorio cumplimiento, se teme a las amonestaciones de tipo legal y económico.

Los sulfuros propios de la etapa de ribera, pueden ser controlados mediante aireación y recirculación, siempre que su concentración no alcance niveles significativos durante el proceso.

El cromo trivalente utilizado se convierte en hidróxido (sales de cromo trivalentes) insoluble en agua, que no forma parte de las cadenas tróficas y por tanto no pueden ser asimilados por las plantas, con la posibilidad de convertirse en cromo hexavalente por oxidación, el cual es más móvil y tóxico.

Los cloruros y sulfatos, son relativamente inofensivos pero las cantidades que se producen salinizan la fuente de agua receptora con altos costos para su remoción posterior.

Algunos de los residuos sólidos pueden ser comercializados y otros requieren de su correcta disposición sin niveles de afectación sentidos en el ambiente, pero aquellos resultantes de los procesos con cromo necesitan ser dispuestos en sitios especialmente diseñados para alojar residuos especiales.

Las industrias de curtiembre, al igual que muchas otras industrias, requieren de una planta para el tratamiento de sus aguas residuales. Sin embargo, teniendo en cuenta que en Colombia la mayoría de ellas son todavía de tipo artesanal, este tipo de solución parece inalcanzable. Las autoridades competentes ante esta situación propusieron la creación de un parque industrial con destino a esta actividad sin que esta oferta haya fructificado hasta la fecha.

En general, en Colombia la industria de las curtiembres se mantiene en un estado latente sobre soluciones ciertas y ejecutables para el manejo de sus residuos sólidos y líquidos. Las industrias pequeñas no disponen de recursos para el tratamiento de sus residuos y la medianas y grandes no son capaces de absorber por sí solas estos costos sin aumentar el precio del producto final y entrar en falta de competitividad con los pequeños productores. Aunque existe preocupación tanto de las autoridades competentes como de los industriales, los estudios realizados por ellos no reflejan con certeza la cantidad y calidad de los vertimientos y por ende para una correcta solución los planes y programas que se planteen deben ser más estrictos que aquellos que podrían iniciarse en otro tipo de condiciones.

En cualquier caso, la afectación ambiental permanece y los recursos continúan agotándose.

\section{Agradecimientos}

El autor desea expresar sus agradecimientos a los ingenieros Sandra Rodríguez y Jorge Amado por su colaboración en el desarrollo de este trabajo.

\section{REFERENCIAS}

[1] ECOPARQUES. (2001). DISEÑO DE UNA PLANTA ESPECIALIZADA DE PELAMBRE Y CURTICIÓN DENTRO DE LAS INSTALACIONES DE COPICUR LTDA PARA BENEFICIO DE LOS CURTIDORES DEL BARRIO SAN BENITO DE BOGOTA, DISTRITO CAPITAL, (SAN BENITO)

[2] CAR. (1982). San Benito, Cundinamarca, Varios expedientes sobre observación de efluentes en curtiembres y otros.

[3] DAMA, (1999). Varios expedientes sobre observación de efluentes en curtiembres, Cundinamarca.

[4] CAR. (1992). Control de contaminación en curtiembres de Villapinzón, aproximación a una solución integral. Cundinamarca. 
[5] BANCO MUNDIAL. (1991). Evaluación Ambiental, Washington D.C.; Volumen II, Número 140.

[6] KEMMER, F. MCCALLION J. (1996) Manual del agua. Naturaleza, tratamiento y aplicaciones. Bogotá, McGraw-Hill, Tomo II, 28.11.

[7] SIERRA CARMONA, J. H. (1997). Metodologías de estudios de impacto ambiental; Predicción y evaluación del impacto en el medio agua. Asociación de Ingenieros Sanitarios de Antioquia, Medellín.

[8] OROZCO JARAMILLO, A. SALAZAR ARIAS A. (1987) Tratamiento biológico de las aguas residuales. Medellín.

[9] RIAÑO, F. ROJAS C. CORREDOR J. (2001). Modelo de contaminación de cursos de aguas naturales. Cuenca Alta del Río Bogotá, Universidad Militar Nueva Granada, Bogotá. 